association was maintained even after adjustments. A cutoff value of 17 for the B-lines score has a sensibility of $73,91 \%$ and a specificity of $69,23 \%$ in identifying patients with $\mathrm{PaO}_{2} / \mathrm{FiO}_{2}<300$.

Conclusion LU can be used for functional lung evaluation and identification of pediatric patients with increase pulmonary water content and decrease $\mathrm{PaO}_{2} / \mathrm{FiO}_{2}$ ratio.

\section{P267 EVALUATION OF A FOCUSED CARDIAC ULTRASOUND PROTOCOL A PEDIATRIC EMERGENCY DEPARTMENT IN VINNITSA REGIONAL CHILDREN HOSPITAL}

Kateryna Dmytriieva, Dmytro Dmytriiev, Oleksandr Katilov*. Vinnitsa National Medical University, Vinnitsa, Ukraine

\subsection{6/archdischild-2019-epa.617}

The objective of this study was to evaluate the implementation of a focused cardiac ultrasound (FoCUS) protocol in a pediatric emergency department (PED).

Methods We conducted a cross-sectional, observational, quality improvement project in a PED of an urban tertiary care Vinnitsa region children hospital. A FoCUS protocol was collaboratively developed by pediatric intensive care and pediatric emergency medicine. This included a reference document with definitions, indications, image acquisition guidelines, and interpretation expectations. We measured physician-emergency pediatric doctors performance against pediatric cardiologist interpretation of stored cine clips as our reference standard. Focused cardiac ultrasound interpretation was dichotomized for the presence or absence of pericardial effusion, depressed left ventricular function, and chamber size abnormalities.

Results 243 FoCUSs were performed by 5 different emergency doctors from January 218 to December 2019. The prevalence of FoCUS abnormalities was 19.4\%. For pericardial effusion, sensitivity was 99,2\% (95\% confidence interval [CI], 48\%$100 \%)$ and specificity was 94\% (95\% CI, 90-100\%). For depressed function, sensitivity was 99,4\% (95\% CI, 56\%$100 \%)$ and specificity was 98\% (95\% CI, 94\%-100\%). For chamber size abnormalities, sensitivity was $97 \% \quad(95 \% \quad \mathrm{CI}$, 50\%-100\%) and specificity was 95\% (95\% CI, 88\%-98\%). The median number of monthly FoCUS increased from 3 (preprotocol) to 7 (postprotocol), and the median rate of adequate studies increased from $0 \%$ to $55 \%$.

Conclusions We report the collaborative development and successful implementation of a PED FoCUS protocol. Physicianemergency doctors interpretation of FoCUS yielded acceptable results.

\section{P268 INTRODUCTION OF THE LOW RISK ANKLE RULE TO A PAEDIATRIC EMERGENCY DEPARTMENT: A QUALITY IMPROVEMENT INITIATIVE}

Peter Tormey, Orla Callender*, Roisin McNamara, Ike Okafor, Patrick Fitzpatrick, Nandini Kandamany, Tracey McCrudden. Children's University Hospital, Temple Street, Dublin, Ireland

\subsection{6/archdischild-2019-epa.618}

Introduction Ankle injuries are common in children $(\sim 2 \%$ of presentations to PEDs) ${ }^{1}$ X-rays are ordered for $85-95 \%$ of patients but only $12 \%$ of $\mathrm{x}$-rays reveal a fracture. ${ }^{1}$ Clinical prediction rules such as The Low Risk Ankle Rule (LRAR) exist to help clinicians safely reduce the frequency of radiography in these injuries. The LRAR has been shown to reduce imaging by up to $60 \%$ without missing any clinically signficant fractures. ${ }^{1}$

Aim To introduce the LRAR to our department and study its effect on our rate of radiography and length of stay (LOS). We also sought to identify complications related to its use, such as missed fractures and representations.

Methods A baseline audit was performed to determine our baseline rate of radiography. The implementation strategy involved: staff education sessions, distribution of study information and visual reminders throughout the department, introduction of mandatory prompts into the x-ray ordering system and a 3 week pilot period.

Results The baseline ankle x-ray rate was 879/969 (90.7\%). This reduced to $40 / 92(43.4 \%)$ using the LRAR, a reduction of $47.3 \%$. The average LOS decreased from 132 minutes before the implementation to 123 minutes after. There were no re-presentations with missed fractures.

Further 12 month follow up data after the initial implementation period showed a persistent $46 \%$ reduction in the frequency of ankle x-rays performed, compared with the baseline data.

Conclusions The LRAR has been shown to safely reduce the frequency of radiography in ankle injuries. ${ }^{1,2}$, Other studies have demonstrated a reduction in x-ray frequency of $22-60 \%$. 1,2 , Our study was in keeping with these previous studies, while also not missing any fractures, further supporting the use of The LRAR in paediatric emergency departments. The LRAR continues to be used effectively in our department. Follow on data showed an annual saving of $€ 28,247$.

\section{REFERENCES}

1. Boutis, K., Von Keyserlingk, C., Willan, A., Narayanan, U. G., Brison, R., Grootendorst, P., ... Goeree, R. (2015). Cost Consequence Analysis of Implementing the Low Risk Ankle Rule in Emergency Departments. Annals of Emergency Medicine, 66, 455-463.e4.

2. Boutis, K., Komar, L., Jaramillo, D., Babyn, P., Alman, B., Snyder, B., ... Schuh, S. (2001). Sensitivity of a clinical examination to predict need for radiography in children with ankle injuries: a prospective study. The Lancet, 358(9299), 2118 2121

\section{P269 IMPROVING THE EMERGENCY DEPARTMENT JOURNEY FOR CHILDREN WITH AUTISM AND COMPLEX NEEDS}

${ }^{1}$ Lisa Dann*, ${ }^{1}$ Peter Tormey, ${ }^{1,2}$ Orla Flanagan. 'University Hospital Galway, Galway, Ireland;

${ }^{2}$ Brother's of Charity, Galway, Ireland

\subsection{6/archdischild-2019-epa.619}

Background Children with autism and complex needs are frequently seen in the Emergency Department (ED). This can be extremely distressing experience for them. This experience can be improved by several measures, including: staff who are experienced in looking after children with autism/complex needs, minimising their time in $\mathrm{ED}$, providing a quiet, relaxing space for them to wait and the use of social stories and play therapists. However, many ED's remain ill-prepared to manage these children.

Aims

- To ascertain staff (paediatric and emergency physicians/nurses) comfort and experience levels in managing children with autism or complex needs in the emergency department. 